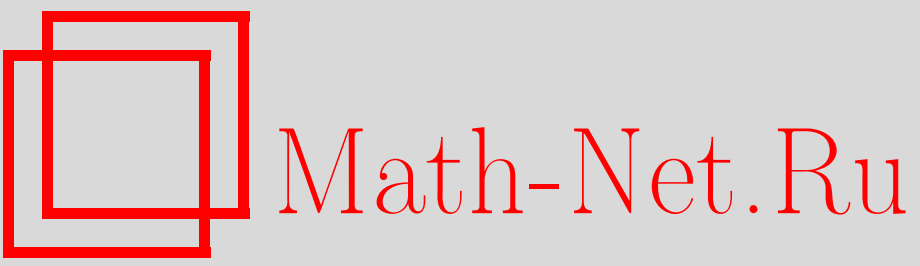

И. В. Зыков, О внешних оценках множеств достижимости управляемых систем с интегральными ограничениями, Итоги науки и техн. Сер. Соврем. мат. и ее прил. Темат. обз., 2021, том 190, 107-114

DOI: https://doi.org/10.36535/0233-6723-2021-190-107-114

Использование Общероссийского математического портала Math-Net.Ru подразумевает, что вы прочитали и согласны с пользовательским соглашением

http: //www.mathnet.ru/rus/agreement

Параметры загрузки:

IP: 34.229 .108 .108

26 апреля 2023 г., 15:37:11 


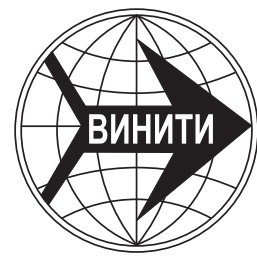

ИТОГИ НАУКИ И ТЕХНИКИ.

Современная математика и ее приложения.

Тематические обзоры.

Том 190 (2021). С. 107-114

DOI: 10.36535/0233-6723-2021-190-107-114

УДК 517.977

О ВНЕШНИХ ОЦЕНКАХ МНОЖЕСТВ ДОСТИЖИМОСТИ УПРАВЛЯЕМЫХ СИСТЕМ С ИНТЕГРАЛЬНЫМИ ОГРАНИЧЕНИЯМИ

(c) 2021 г. $\quad$ И. В. ЗЫКОВ

\begin{abstract}
АннотАция. В работе рассматривается задача построения внешних оценок множеств достижимости в виде множества уровня некоторой дифференцируемой функции Ляпунова-Беллмана для управляемой системы с интегральным ограничением на управление из $\mathbb{L}_{p}, p>1$. При удачном ее выборе можно получить эллипсоидальные и прямоугольные оценки. Предлагаемые построения основаны на интегральных оценках, максимальном решении и принципе сравнения для систем дифференциальных неравенств. За счет использования времени в аргументах функции Ляпунова-Беллмана удается получить более точные оценки. В линейном нестационарном случае последние могут совпадать с множеством достижимости. Приведены иллюстрирующие примеры для нелинейных систем.
\end{abstract}

Ключевые слова: множество достижимости, управляемая система, интегральное ограничение, интегральное неравенстве, принцип сравнения, внешняя оценка.

\title{
EXTERNAL ESTIMATES OF REACHABLE SETS \\ FOR CONTROL SYSTEMS WITH INTEGRAL CONSTRAINTS
}

\author{
(c) 2021 I. V. ZYKOV
}

\begin{abstract}
In this paper, we consider the problem of constructing external estimates for reachable sets as level sets of some differentiable Lyapunov-Bellman function for a control system with an integral control constraint from $\mathbb{L}_{p}, p>1$. For an appropriate choice of this function, ellipsoidal and rectangular estimates can be obtained. Constructions proposed are based on integral estimates, maximal solutions, and the comparison principle for systems of differential inequalities. Using time as an argument of the Lyapunov-Bellman function, we obtain more accurate estimates. In the linear nonstationary case, such estimates may coincide with the reachable set. Also we present illustrative examples for nonlinear systems.
\end{abstract}

Keywords and phrases: reachable set, control system, integral constraint, integral inequality, comparison principle, external estimate.

AMS Subject Classification: 49-XX

1. Введение. Статья посвящена построению внешних оценок для множеств достижимости управляемых систем. Аналитические построения последних часто не представляются возможными, а их численное построение требует больших вычислительных затрат. В отдельных задачах достаточно получить только оценки (внешние или внутренние) для множеств достижимости, которые менее трудоемки по сравнению с построением самих множеств. Для систем с геометрическими ограничениями на управление известны методы, основанные на оценках решений дифференциальных неравенств Гамильтона-Якоби и Ляпунова-Кротова (см., например, [1-3,5,7]). Методы данного типа основаны на вычислении производной вдоль траектории управляемой системы 
от некоторой дифференцируемой функции (функции Ляпунова-Беллмана), максимизации величины производной на множестве управляющих параметров и применении принципа сравнения для дифференциальных уравнений.

В первой части данной работы предлагается способ построения внешних оценок для нелинейных управляемых систем с интегральным ограничением на управление из $\mathbb{L}_{p}, p>1$ с помощью множества уровня функций Ляпунова-Беллмана, зависящих только от вектора состояния системы. Во второй части работы обсуждается возможность улучшения оценок путем добавления времени в число аргументов функции Ляпунова-Беллмана. Зависимость функции ЛяпуноваБеллмана от времени позволяет получать более точные оценки. В линейно-квадратичном случае оценка может совпасть с множеством достижимости (при условии, что начальное состояние фиксировано).

Полученные ниже уравнения сравнения могут иметь не единственное решение. Это требует привлечения понятия максимального (верхнего) решения уравнения и теорем сравнения, использующих данное понятие.

Рассмотрим задачу Коши

$$
\frac{d x}{d t}=F(t, x), \quad x\left(t_{0}\right)=x_{0},
$$

где $F(t, x)$ - непрерывная функция на $(t, x)$-множестве $D_{o}$.

Определение 1. Решение $G(t)=G\left(t ; t_{0}, x_{0}\right)$ дифференциального уравнения (1), выходящее из точки $\left(t_{0}, x_{0}\right)$ области $D_{o}^{*} \subset D_{o}$ и определенное на некотором отрезке $[a, b]$, содержащем внутри точку $t_{0}$, будем называть верхним (максимальным), если для любого другого решения, выходящего из этой точки, неравенство $\varphi(t) \leqslant G(t)$ справедливо для всех $t \in[a, b]$, для которых решения $\varphi(t)$ и $G(t)$ определены одновременно.

Условия, обеспечивающие существование верхних решений (локальных и глобальных), содержатся в ряде известных теорем (см., например, [6, с. 7-8]), которые здесь приводить не будем.

Следующая теорема будет играть ключевую роль при построении функций Ляпунова-Беллмана.

Теорема 1 (теорема сравнения, [6, с. 10-11]). Пусть функиия $F(t, z)$ непрерывна в открытой области $D_{o}$, содержащей точку $\left(t_{0}, x_{0}\right)$. Предположим, что начальная задача

$$
\frac{d z}{d t}=F(t, z), \quad z\left(t_{0}\right)=z_{0}
$$

имеет верхнее решение $z(t)=G(t)$, определенное в области $t_{0} \leqslant t \leqslant t_{0}+\delta$. Eсли $x(t)-$ такая непрерывная и дифферениируемая на $\left[t_{0}, t_{0}+\delta\right]$ функиия, что $(t, x(t)) \in D_{o} \partial л_{я}$ всех $t \in\left[t_{0}, t_{0}+\delta\right] u$

то справедлива ощенка

$$
\frac{d x}{d t} \leqslant F(t, x(t)) \quad \forall t \in\left[t_{0}, t_{0}+\delta\right], \quad x\left(t_{0}\right) \leqslant z_{0},
$$

$$
x(t) \leqslant G(t) \quad \forall t \in\left[t_{0}, t_{0}+\delta\right] .
$$

2. Внешние оценки и принцип сравнения. Рассмотрим автономную линейную по управлению систему вида

$$
\dot{x}(t)=f_{1}(x(t))+f_{2}(x(t)) u(t), \quad 0 \leqslant t \leqslant t_{1}, \quad x(0)=x^{0},
$$

где $x \in \mathbb{R}^{n}$ - вектор состояния; $u(t) \in \mathbb{R}^{r}$ - управление; $f_{1}: \mathbb{R}^{n} \rightarrow \mathbb{R}^{n}, f_{2}: \mathbb{R}^{n} \rightarrow \mathbb{R}^{n \times r}$ - непрерывные отображения.

Интегральное ограничение на управление имеет вид $u(\cdot) \in U$, где

$$
U=\left\{u(\cdot) \in \mathbb{L}_{p}: \int_{0}^{t_{1}}\|u(t)\|_{r}^{p} d t \leqslant \mu^{p}\right\}, \quad p>1, \quad \mu>0 .
$$

Определение 2. Множеством достижимости $K(t)$ системы (2) в момент $t \leqslant t_{1}$ будем называть совокупность всех концов траекторий $x(t)$ в $\mathbb{R}^{n}$, отвечающих управлениям из $U$. 
Пусть известно множество $D \subset \mathbb{R}^{n}$, которое содержит точки всех допустимых траекторий, удовлетворяющих заданному начальному условию и интегральному ограничению на управление. Рассмотрим такую непрерывно дифференцируемую функцию $v(x)$ на $D$, что $v\left(x^{0}\right)=0$.

Предположение 1. Пусть на множестве $D$ выполняются неравенства

$$
\nabla v(x) f_{1}(x) \leqslant-\alpha v(x), \quad\left\|\nabla v(x) f_{2}(x)\right\|_{r}^{q} \leqslant \beta^{q} v(x), \quad \frac{1}{p}+\frac{1}{q}=1,
$$

где $\alpha \geqslant 0$ и $\beta>0$ - заданные константы, а через $\nabla v(x)$ обозначен градиент функции $v$.

Заметим, что из второго условия (неравенства) следует неотрицательность $v(x)$.

Теорема 2. Пусть выполнено предположение 1. Тогда

$$
K(t) \subset\left\{x \in \mathbb{R}^{n}: v(x) \leqslant \psi(t)\right\}, \quad t \in\left[0, t_{1}\right],
$$

где $\psi(t)=p(\mu \beta / p)^{p} t^{p-1}$ в случае $\alpha=0$. В случае $\alpha>0$ можно положить

$$
\psi(t)=\alpha\left(\frac{\mu \beta}{\alpha}\right)^{p}\left(1-\exp \left(-\frac{\alpha}{p} t\right)\right)^{p-1},
$$

либо (что дает более точную оченку)

$$
\psi(t)= \begin{cases}\frac{\mu^{2} \beta^{2}}{\alpha}\left(1-\exp \left(-\frac{\alpha}{2} t\right)\right) \exp \left(-\frac{\alpha}{2} t\right), & \text { если } t<\frac{p \ln p}{\alpha}, \\ \frac{\alpha}{p-1}\left(\frac{\mu \beta}{\alpha}\right)^{p}\left(\frac{p-1}{p}\right)^{p}, & \text { если } t \geqslant \frac{p \ln p}{\alpha} .\end{cases}
$$

Доказательство. Дифференцируя $v(x(t))$ вдоль допустимой траектории $x(t)$, получим

$$
\dot{v}(x(t))=\nabla v(x(t))\left(f_{1}(x(t))+f_{2}(x(t)) u(t)\right)=\nabla v(x(t)) f_{1}(x(t))+\nabla v(x(t)) f_{2}(x(t)) u(t),
$$

откуда следует, что

$$
\begin{gathered}
v(x(t))=\int_{0}^{t} \nabla v(x(s)) f_{1}(x(s)) d s+\int_{0}^{t} \nabla v(x(s)) f_{2}(x(s)) u(s) d s \\
v(x(t)) \leqslant-\alpha \int_{0}^{t} v(x(s)) d s+\left(\int_{0}^{t}\|u(s)\|_{r}^{p} d s\right)^{1 / p}\left(\int_{0}^{t}\left\|\nabla v(x(s)) f_{2}(x(s))\right\|_{r}^{q} d s\right)^{1 / q}, \\
v(x(t)) \leqslant-\alpha \int_{0}^{t} v(x(s)) d s+\mu \beta\left(\int_{0}^{t} v(x(s)) d s\right)^{1 / q} .
\end{gathered}
$$

Последняя оценка эквивалентна неравенству

$$
\dot{f}(t) \leqslant-\alpha f(t)+\mu \beta \cdot f(t)^{1 / q},
$$

если обозначить

$$
f(t)=\int_{0}^{t} v(x(s)) d s .
$$

Поставим в соответствие этому неравенству уравнение сравнения

$$
\dot{w}=-\alpha w+\mu \beta \cdot w^{1 / q}
$$

с начальным условием $w(0)=f(0)=0$.

Пусть $\alpha=0$ в уравнении (4). Интегрируя, получим два решения:

$$
w(t)=0 \quad \text { и } \quad w(t)^{1 / p}=\frac{\mu \beta}{p} t, \quad t \in\left[0, t_{1}\right] .
$$


Второе из них является, очевидно, максимальным. Из теоремы 1 получаем при $t \in\left[0, t_{1}\right]$ неравенство

$$
f(t) \leqslant \bar{w}(t)=\left(\frac{\mu \beta}{p}\right)^{p} t^{p},
$$

где $\bar{w}(t)$ - верхнее решение (4). Так как из неравенства (3) следует, что

$$
v(x(t)) \leqslant \mu \beta \cdot f(t)^{1 / q}=\mu \beta \cdot f(t)^{(p-1) / p},
$$

то в этом случае

$$
v(x(t)) \leqslant \psi(t)=p\left(\frac{\mu \beta}{p}\right)^{p} t^{p-1} .
$$

При $\alpha>0$, используя, например, подстановку вида $y=w^{1 / p}$ в уравнении сравнения (4), приходим к линейному уравнению

$$
\dot{y}=-\frac{\alpha}{p} y+\frac{\mu \beta}{p}, \quad y(0)=w(0)^{1 / p}=0 .
$$

В силу линейности последнее имеет единственное решение, которое совпадает с верхним или максимальным решением. Поэтому максимальное решение выглядит следующим образом:

$$
\bar{w}(t)^{1 / q}=\left(\frac{\mu \beta}{\alpha}\right)^{p-1}\left(1-\exp \left(-\frac{\alpha}{p} t\right)\right)^{p-1}, \quad t \in\left[0, t_{1}\right] .
$$

Учитывая цепочку неравенств

$$
v(x(t)) \leqslant-\alpha f(t)+\mu \beta \cdot f(t)^{1 / q} \leqslant \mu \beta \cdot f(t)^{1 / q},
$$

по аналогии со случаем $\alpha=0$ получаем, что

$$
v(x(t)) \leqslant \psi(t)=\alpha\left(\frac{\mu \beta}{\alpha}\right)^{p}\left(1-\exp \left(-\frac{\alpha}{p} t\right)\right)^{p-1} .
$$

Последнюю оценку можно улучшить, если в качестве $\psi(t)$ взять

$$
\psi(t)=\max _{0 \leqslant f \leqslant \bar{w}(t)}\left\{-\alpha f+\mu \beta f^{\frac{1}{q}}\right\} .
$$

Вычисляя максимум, получим, что

$$
\psi(t)=\frac{\alpha}{p-1}\left(\frac{\mu \beta}{\alpha}\right)^{p}\left(\frac{p-1}{p}\right)^{p}, \quad p>1,
$$

если

$$
\left(\frac{\mu \beta}{\alpha}\right)^{p}\left(\frac{p-1}{p}\right)^{p} \leqslant \bar{w}(t)
$$

что эквивалентно следующим неравенствам:

$$
\left(1-\frac{1}{p}\right)^{p} \leqslant\left(1-\exp \left(-\frac{\alpha}{p} t\right)\right)^{p}, \quad \exp \left(-\frac{\alpha}{p} t\right) \leqslant \frac{1}{p}, \quad t \geqslant \frac{p \ln p}{\alpha} .
$$

В противном случае

$$
\psi(t)=-\alpha \bar{w}(t)+\mu \beta \bar{w}(t)^{1-\frac{1}{p}}=\frac{\mu^{2} \beta^{2}}{\alpha}\left(1-\exp \left(-\frac{\alpha}{2} t\right)\right) \exp \left(-\frac{\alpha}{2} t\right)
$$

(не выполняется (5)). Таким образом, для любого $t \leqslant t_{1}$ мы имеем оценку сверху для множества достижимости вида

$$
K(t) \subset\left\{x \in \mathbb{R}^{n}: v(x) \leqslant \psi(t)\right\},
$$

где функция $\psi(t)$ определяется одной из приведенных выше формул. 
3. Нестационарный случай. Если рассматривать функции Ляпунова-Беллмана, зависящие от $t, x$, то можно получить более точные оценки даже в случае стационарных систем. Проиллюстрируем это утверждение на примере линейных систем с интегральными ограничениями.

Вначале рассмотрим линейную систему с интегральным ограничением на управление и фиксированным начальным состоянием

$$
\dot{x}(t)=A(t) x(t)+B(t) u(t), \quad t \in\left[t_{0}, t_{1}\right], \quad x\left(t_{0}\right)=x^{0},
$$

где $x \in \mathbb{R}^{n}, u(t) \in \mathbb{R}^{r} ; A(t), B(t)$ интегрируемые на $\left[t_{0}, t_{1}\right]$ матричные функции. Ограничение на управление определено интегральным квадратичным неравенством $u(\cdot) \in U$, где

$$
U=\left\{u(\cdot) \in \mathbb{L}_{2}: \int_{t_{0}}^{t_{1}}\|u(t)\|_{r}^{2} d t \leqslant \mu^{2}\right\}, \quad \mu>0 .
$$

Пусть $X(t, s)=\Phi(t) \Phi^{-1}(s)$, где $\Phi(t)$ - матрица Коши, удовлетворяющая уравнению

$$
\dot{\Phi}(t)=A(t) \Phi(t), \quad \Phi\left(t_{0}\right)=I .
$$

Решение (6) имеет вид

$$
x(t)=\hat{x}(t)+\int_{t_{0}}^{t} X(t, s) B(s) u(s) d s,
$$

где

$$
\hat{x}(t)=X\left(t, t_{0}\right) x^{0}
$$

Симметрическую матрицу

$$
W(t)=\int_{t_{0}}^{t} X(t, s) B(s) B^{\top}(s) X^{\top}(t, s) d s
$$

будем называть грамианом управляемости системы (6). Данная матрица удовлетворяет следующему матричному дифференциальному уравнению:

$$
\dot{W}(t)=A(t) W(t)+W(t) A^{\top}(t)+B(t) B^{\top}(t), \quad W\left(t_{0}\right)=0 .
$$

Как известно (см., например, [9]), система (6) вполне управляема на $\left[t_{0}, t\right], t \leqslant t_{1}$, тогда и только тогда, когда матрица $W(t)$ положительно определена. В этом случае $K(t)$ представляет собой невырожденный эллипсоид

$$
K(t)=\left\{x \in \mathbb{R}^{n}:(x-\hat{x})^{\top} W^{-1}(t)(x-\hat{x}) \leqslant \mu^{2}\right\}, \quad t>t_{0} .
$$

Непрерывно дифференцируемую по своим аргументам функцию $v: \mathbb{R}^{n+1} \rightarrow \mathbb{R}$ будем искать в виде положительно определенной квадратичной формы

$$
v(t, x-\hat{x})=(x-\hat{x})^{\top} Q(t)(x-\hat{x}) \geqslant 0, \quad Q^{\top}=Q .
$$

Рассмотрим следующее дифференциальное уравнение Риккати для матрицы $Q(t)$ :

$$
\dot{Q}(t)+Q(t) A(t)+A^{\top}(t) Q(t)=-Q(t) B(t) B^{\top}(t) Q(t) .
$$

Теорема 3. Пусть существует положительно определенное на $\left[t_{0}, t_{1}\right]$ решение уравнения (10). Тогда для множества достижсиости системы (6) справедлива оченка

$$
K\left(t_{1}\right) \subset\left\{x \in \mathbb{R}^{n}: v\left(t_{1}, x\right)=\left(x-\hat{x}\left(t_{1}\right)\right)^{\top} Q\left(t_{1}\right)\left(x-\hat{x}\left(t_{1}\right)\right) \leqslant \mu^{2}\right\}, \quad t_{1}>t_{0} .
$$


Доказательство. Продифференцируем $v$ вдоль траекторий управляемой системы (6):

$$
\begin{aligned}
& \begin{array}{r}
\dot{v}_{t}=(\dot{x}-\dot{\hat{x}})^{\top} Q(t)(x-\hat{x})+(x-\hat{x})^{\top} \dot{Q}(t)(x-\hat{x})+(x-\hat{x})^{\top} Q(t)(\dot{x}-\dot{\hat{x}})= \\
=\left((x-\hat{x})^{\top} A^{\top}(t)+u^{\top} B^{\top}(t)\right) Q(t)(x-\hat{x})+(x-\hat{x})^{\top} \dot{Q}(t)(x-\hat{x})+ \\
\quad+(x-\hat{x})^{\top} Q(t)(A(t)(x-\hat{x})+B(t) u)= \\
=(x-\hat{x})^{\top}\left(\dot{Q}(t)+Q(t) A(t)+A^{\top}(t) Q(t)\right)(x-\hat{x})+2(x-\hat{x})^{\top} Q(t) B(t) u .
\end{array}
\end{aligned}
$$

Учитывая (10), равенство (11) можно преобразовать к виду

$$
\dot{v}_{t}=-(x-\hat{x})^{\top} Q(t) B(t) B^{\top}(t) Q(t)(x-\hat{x})+2(x-\hat{x})^{\top} Q(t) B(t) u=-f(t) f^{\top}(t)+2 f(t) u,
$$

где $f(t)=(x-\hat{x})^{\top} Q(t) B(t)$. Отсюда следует неравенство для функции $v$ :

$$
\begin{aligned}
& v(t, x-\hat{x})=-\int_{t_{0}}^{t}\|f(s)\|_{r}^{2} d s+2 \int_{t_{0}}^{t} f(s) u(s) d s \leqslant \\
& \leqslant-\int_{t_{0}}^{t}\|f(s)\|_{r}^{2} d s+2 \mu\left(\int_{t_{0}}^{t}\|f(s)\|_{r}^{2} d s\right)^{1 / 2}=-\gamma^{2}(t)+2 \mu \gamma(t)=\mu^{2}-(\gamma(t)-\mu)^{2} \leqslant \mu^{2} .
\end{aligned}
$$

где

$$
\gamma(t)=\left(\int_{t_{0}}^{t}\|f(s)\|_{r}^{2} d s\right)^{1 / 2} .
$$

Таким образом, имеем оценку сверху для множества достижимости вида

$$
K\left(t_{1}\right) \subset\left\{x \in \mathbb{R}^{n}: v\left(t_{1}, x\right)=\left(x-\hat{x}\left(t_{1}\right)\right)^{\top} Q\left(t_{1}\right)\left(x-\hat{x}\left(t_{1}\right)\right) \leqslant \mu^{2}\right\}, \quad t_{1}>t_{0} .
$$

Теорема доказана.

Замечание 1. Дифференцируя равенство $W^{-1}(t) W(t)=I$, с учетом (9) получаем

$$
\begin{gathered}
\dot{W}^{-1}(t) W(t)+W^{-1}(t) \dot{W}(t)=0 \\
\dot{W}^{-1}(t)+W^{-1}(t) A(t)+A^{\top}(t) W^{-1}(t)=-W^{-1}(t) B(t) B^{\top}(t) W^{-1}(t), \quad t>t_{0} .
\end{gathered}
$$

Таким образом, $W^{-1}(t)$ является решением уравнения Риккати $(10)$. Если в качестве $Q(t)$ взять $W^{-1}(t)$, то оценка (13) совпадает с множеством достижимости. Однако заметим, что $W\left(t_{0}\right)=0$ и, следовательно, $W^{-1}(t)$ не определена при $t=t_{0}$. Кроме того, обращение $W(t)$ возможно, если система вполне управляема на любом отрезке $\left[t_{0}, t\right]$. Последнее условие выполняется по умолчанию для стационарных систем в предположении полной управляемости.

Расширим результат данного раздела на случай, когда начальный вектор состояния принадлежит заданному множеству. Рассмотрим управляемую систему (6) при условии, что $x^{0} \in X^{0}$, где $X^{0}$ - компактное множество из $\mathbb{R}^{n}$, а интегральное ограничение на управляющее воздействие имеет такой же вид (7).

Непрерывно дифференцируемую по своим аргументам функцию $V: \mathbb{R}^{n+1} \rightarrow \mathbb{R}$ будем искать в виде $V(t, y)=v(t, y)-v\left(t_{0}, y\left(t_{0}\right)\right)$, где $v(t, y)=y^{\top} Q(t) y, y=x-\hat{x}, Q^{\top}=Q \geqslant 0$. Здесь, в отличие от (8), центр

$$
\hat{x}(t)=X\left(t, t_{0}\right) \bar{x}, \quad \bar{x} \in \mathbb{R}^{n} .
$$

В этом случае в силу (6) и (15)

$$
\dot{y}(t)=A(t) y(t)+B(t) u(t), \quad t \in\left[t_{0}, t_{1}\right], \quad y\left(t_{0}\right)=x^{0}-\bar{x} .
$$




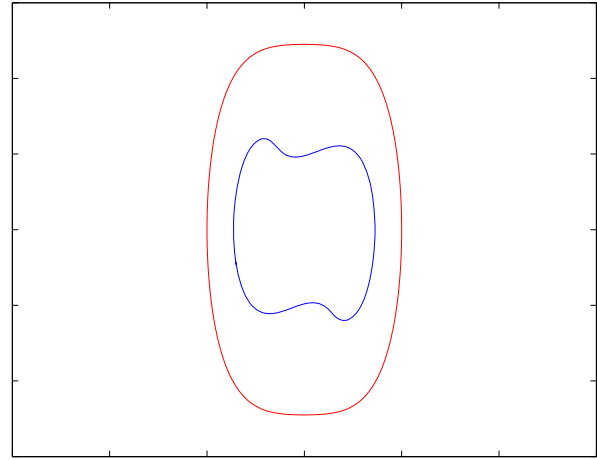

Рис. 1. Граница множества достижимости системы (16) и ее оценка

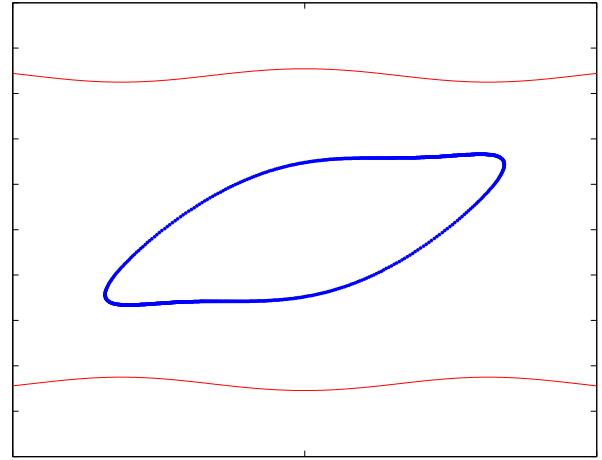

Рис. 2. Граница множества достижимости системы (17) и ее оценка

Далее, следуя схеме доказательства теоремы 3, можно получить внешнюю оценку для множества достижимости

$K\left(t_{1}\right) \subset\left\{x \in \mathbb{R}^{n}: v\left(t_{1}, x\right)=\left(x-\hat{x}\left(t_{1}\right)\right)^{\top} Q\left(t_{1}\right)\left(x-\hat{x}\left(t_{1}\right)\right) \leqslant \mu^{2}+\min _{\bar{x} \in \mathbb{R}^{n}} \max _{x^{0} \in X^{0}} v\left(t_{0}, x^{0}-\bar{x}\right)\right\}, \quad t_{1}>t_{0}$.

\section{4. Примеры.}

Пример 1 (осциллятор Дуффинга). Рассмотрим осциллятор Дуффинга, описываемый уравнениями

$$
\dot{x}_{1}=x_{2}, \quad \dot{x}_{2}=-x_{1}-10 x_{1}^{3}+u, \quad x(0)=0, \quad t \in[0,3 / 2],
$$

с интегральным ограничением на управление

$$
\int_{0}^{3 / 2} u^{2}(t) d t \leqslant 2 .
$$

За функцию $v(x)$ можно принять

$$
v\left(x_{1}, x_{2}\right)=\frac{5}{2} x_{1}^{4}+\frac{1}{2} x_{1}^{2}+\frac{1}{2} x_{2}^{2}
$$

она удовлетворяет предположению 1 с параметрами $\alpha=0, \beta^{2}=2$ и $\mu^{2}=2$. В этом случае

$$
K(3 / 2) \subset\left\{x \in \mathbb{R}^{2}: v\left(x_{1}, x_{2}\right) \leqslant \psi(3 / 2)=3\right\} .
$$

Результат представлен на рис. 1, где синим цветом обозначена граница множества достижимости (полученная при помощи алгоритма из [8]), а красным - его оценка.

Пример 2 (математический маятник). Рассмотрим математический маятник с ограничением на энергию управления:

$$
\dot{x}_{1}=x_{2}, \quad \dot{x}_{2}=-\frac{1}{2} \sin x_{1}+u, \quad x(0)=0, \quad t \in[0, \pi],
$$

с интегральным ограничением на управление

$$
\int_{0}^{\pi} u^{2}(t) d t \leqslant 2
$$

Функция $v(x)$ может иметь вид

$$
v\left(x_{1}, x_{2}\right)=\frac{1}{2}\left(1-\cos x_{1}\right)+\frac{1}{2} x_{2}^{2}
$$


эта функция удовлетворяет предположению 1 с параметрами $\alpha=0, \beta^{2}=2$ и $\mu^{2}=2$. В этом случае

$$
K(\pi) \subset\left\{x \in \mathbb{R}^{2}: v\left(x_{1}, x_{2}\right) \leqslant \psi(\pi)=2 \pi\right\} .
$$

Результат численного моделирования представлен на рис. 2. В данном случае видно, что оценка (красный цвет) в виде бесконечной волнистой полосы не дает информации по координате $x_{1}$ для границы множества достижимости (синий цвет).

Данную проблему можно решить, используя первое уравнение из (17) и полученную оценку:

$$
\begin{gathered}
\left|x_{1}(t)\right| \leqslant \int_{0}^{t}\left|x_{2}(s)\right| d s \leqslant\left(\int_{0}^{t} 1 d s\right)^{1 / 2}\left(\int_{0}^{t} x_{2}^{2}(s) d s\right)^{1 / 2}=\sqrt{t}\left(\int_{0}^{t} x_{2}^{2}(s) d s\right)^{1 / 2}, \\
\frac{1}{2}\left(1-\cos x_{1}(t)\right)+\frac{1}{2} x_{2}^{2}(t) \leqslant \psi(t)=2 t, \quad x_{2}^{2}(t) \leqslant 4 t-1+\cos x_{1}(t) \leqslant 4 t .
\end{gathered}
$$

Отсюда заключаем, что $\left|x_{1}(t)\right| \leqslant \sqrt{2} t^{3 / 2}, t \in[0, \pi]$. Граница множества достижимости, изображенная на рис. 2 синим цветом, построена с помощью алгоритма из [4].

\section{СПИСОК ЛИТЕРАТУРЫ}

1. Гурман В. И. Принцип расширения в задачах управления. - М.: Физматлит, 1997.

2. Гусев М. И. О внешних оценках множеств достижимости нелинейных управляемых систем// Тр. Ин-та мат. мех. УрО РАН. - 2011. - 17, № 1. - С. 60-69.

3. Дыхта В. А. Неравенство Ляпунова--Кротова и достаточные условия в оптимальном управлении// Итоги науки и техн. Сер. Соврем. мат. прилјож. Темат. обзоры. - 2006. - 110. - C. 76-108.

4. Зыков И. В. О задаче достижимости для нелинейной управляемой системы с интегральными ограничениями// CEUR-WS Proc. - 2017. - 1894. - C. 88-97.

5. Куржанский А. Б. Принцип сравнения для уравнений типа Гамильтона-Якоби в теории управления// Тр. Ин-та мат. мех. УрО РАН. - 2006. - 12, № 1. - С. 173-183.

6. Мартынюк А. А., Лакшмикантам В., Лила С. Устойчивость движения: метод интегральных неравенств. - Киев: Наукова думка, 1989.

7. Никольский M. С. Об оценивании множества достижимости для некоторых управляемых объектов// Мат. Междунар. конф., посв. 110-летию со дня рождения Л. С. Понтрягина. - М., 2018. - С. 194-196.

8. Gusev M. I., Zykov I. V. On extremal properties of boundary points of reachable sets for a system with integrally constrained control// IFAC-PapersOnLine. - 2017. - 50. — P. 4082-4087.

9. Lee E. B., Marcus L. Foundations of Optimal Control Theory. — New York: Wiley, 1967.

Зыков Игорь Владимирович

Институт математики и механики им. Н. Н. Красовского

Уральского отделения РАН, Екатеринбург;

Уральский федеральный университет

им. первого Президента России Б. Н. Ельцина, Екатеринбург

E-mail: zykoviustu@mail.ru 